\title{
Estimación de la edad de Swietenia macrophylla (Meliaceae) a partir del diámetro normal en poblaciones del sureste mexicano
}

\author{
Jonathan Hernández-Ramos ${ }^{1,2}$, Juan Ignacio Valdez-Hernández², Xavier García-Cuevas ${ }^{1}$,
} Alfredo Esteban Tadeo-Noble ${ }^{2} \&$ Valentín José Reyes-Hernández ${ }^{1}$

1. Instituto Nacional de Investigaciones Forestales, Agrícolas y Pecuarias (INIFAP). Campo Experimental Chetumal. Carretera Chetumal-Bacalar Km 25, Xul-Ha, Othón P. Blanco, 77930, Quintana Roo, México; forestjonathanhdez@gmail.com, garcia.xavier@inifap.gob.mx

2. Colegio de Postgraduados (COLPOS). Campus Montecillo. Carretera México-Texcoco Km. 36.5, Montecillo, Texcoco, 56230, Estado de México, México; ignaciov@colpos.mx, alfredotadeo@gmail.com, vreyes@colpos.mx

Recibido 19-VI-2019. Corregido 09-IX-2019. ～Aceptado 23-II-2019.

\begin{abstract}
Age estimation of Swietenia macrophylla (Meliaceae) from the normal diameter in populations of Southeastern Mexico. Introduction: Swietenia macrophylla King (Mahogany) is the most commercially important tree species in Southeastern of Mexico and is used as a guide in forest management. Therefore, knowing its age and growth rates is essential for the sustainability of timber harvest. Objective: Estimate the age $(E t)$ from the 'step time' $(T p)$ method and calculate the absolute $(T C A)$ and relative $(T C R)$ growth rates for Swietenia macrophylla in Quintana Roo, Mexico. Methods: Equations were adjusted to calculate TCA and $T C R$ with periodic re-measured for 15 years of the normal diameter $(d)$ in 403 S. macrophylla trees in Felipe Carrillo Puerto, Quintana Roo, Mexico. Tp and Et were determined for the calculation of the annual current increase $(I C A)$ and average annual increase $(I M A)$, to define the commercial and technical shifts. Results: The maximum $T C R$ was reached at $28 \mathrm{~cm}$ of $d\left(0.015 \mathrm{~cm}_{\text {year }}{ }^{-1}\right)$ (thinning application), whereas the highest $T C A$ $\left(0.69 \mathrm{~cm} \mathrm{year}^{-1}\right)$ and lower $T p$ (3.5 years) at $55 \mathrm{~cm}$ of $d$ (commercial shift). The maximum ICA when considering Et was $1.43 \mathrm{~cm}$ in the $50 \mathrm{~cm}$ of diametric category with an IMA of $0.67 \mathrm{~cm}_{\text {year }}{ }^{-1}$ at an age of 75 years, while the technical shift $\left(\right.$ ICA $=$ IMA) was reached at 96 years (increments of $0.78 \mathrm{~cm}^{-1}$ year $\left.^{-1}\right)$. Conclusions: Through periodic measurements of the normal diameter in S. macrophylla it is possible to obtain its age, these results also can be used to elaborate management programs and apply silvicultural practices that propitiate the diametric increase in Quintana Roo.
\end{abstract}

Key words: timber harvest, mahogany, growth rates, passage time.

Hernández-Ramos, J., Valdez-Hernández, J. I., García-Cuevas, X., Tadeo-Noble, A. E., \& Reyes-Hernández, V. J. (2020). Estimación de la edad de Swietenia macrophylla (Meliaceae) a partir del diámetro normal en poblaciones del sureste mexicano. Revista de Biología Tropical, 68(1), 200-217.

En el manejo de los bosques, conocer el incremento, crecimiento, turno técnico y rendimiento del arbolado es fundamental para la planeación de actividades silvícolas acorde a las condiciones actuales de la masa forestal (Clutter, Forston, Pienaar, Brister, \& Bailey, 1983; Domínguez-Calleros, Rodríguez-Flores,
Lizárraga-Mendiola, Jiménez-Gómez, \& Navar, 2017). El crecimiento es el aumento en las dimensiones de un árbol, rodal o bosque en un lapso de tiempo, mientras que el rendimiento es la acumulación del crecimiento por unidad de superficie a una edad establecida (Prodan, Peters, Cox, \& Real, 1997), ambos 
influenciados por la información genética de la especie, el ambiente y la interacción de ambos (Smith, Leemans, \& Shugart, 1992; Purata, Gerez, \& Peters, 2016).

Por otro lado, el turno técnico se define como el momento en el cual los incrementos corriente y medio anual se igualan (ICA = IMA), e indica la edad de rotación de la especie (Imaña \& Encinas, 2008; Bonilla \& Alarcón, 2015). El turno comercial es el número de años necesario para que un árbol tenga las dimensiones que demanda la industria del aserrío (Dykstra \& Heinrich, 1996; Casals, 2005). La combinación de la edad con la información silvícola cuantitativa, es la base para realizar aclareos como tratamiento silvícola que trata de aprovechar el potencial de crecimiento de las especies a través de la redistribución del arbolado al eliminar algunos individuos y controlar la densidad (Musálem, 1989; Meza \& Torres, 2006).

Determinar la edad de los árboles en los bosques tropicales es complejo, debido a la ausencia de anillos de crecimiento definidos, a las malformaciones en éstos cuando están presentes o a la falta de visibilidad por el desarrollo continuo del individuo (Del Valle, 1986, 1997; López-Ayala, Valdez-Hernández, Terrazas, \& Valdez-Lazalde, 2006a; López-Hernández et al., 2018). También, influye la disminución del diámetro normal entre periodos de tiempo cortos debido a condiciones ambientales adversas para su crecimiento, como el estrés hídrico por la baja disponibilidad de agua (Pineda-Herrera, Valdez-Hernández, \& López-López, 2012) o una evapo-transpiración alta por temperaturas elevadas (Reich \& Borchert, 1982; Baker, Affum-Baffoe, Burslem, \& Swaine, 2002; López-Ayala, Valdez-Hernández, Terrazas, \& Valdez-Lazalde, 2006b).

Algunas técnicas para estimar la edad en especies sin anillos de crecimiento visibles o definidos, son las heridas en el cambium vascular (Pineda-Herrera, Valdez-Hernández, López-López, Manzano-Méndez, \& SalgadoUgarte, 2015a), el contenido de Carbono-14 $\left({ }^{14} \mathrm{C}\right)$ (Del Valle, 1997; Hart, 2010), el uso de ecuaciones de crecimiento e incremento basadas en el diámetro (Del Valle, 1986; Ricker \& Del Río, 2004) y el tiempo que tarda una especie en pasar de una categoría diamétrica (CD) a otra (i.e. tiempo de paso) (LópezTorres \& Tamarit-Urias, 2005). Sin embargo, en muchas ocasiones solo se calcula la velocidad de incremento a través del diámetro normal en dos periodos de tiempo distintos $\left(d_{2}-d_{1}\right)$ (Manzano-Méndez, Valdez-Hernández, LópezLópez, \& Vaquera-Huerta, 2010; Interián-Ku et al., 2014; Domínguez-Calleros et al., 2017), sin llegar a determinar la edad de los individuos. El diámetro normal es la variable más utilizada en el levantamiento de información en inventarios forestales en el mundo (Van Laar \& Akça, 1997; Prodan et al., 1997). Con esta variable se puede determinar la edad esperada en los árboles en los bosques tropicales, a través del incremento corriente a un diámetro establecido (Del Valle, 1986, 1997) o en un lapso de tiempo determinado (Hernández \& Castellanos, 2006; Domínguez-Calleros et al., 2017).

En México, conocer tanto la tasa de crecimiento como la edad de S. macrophylla King (Caoba) es fundamental para su manejo, ya que es la especie más importante en el aprovechamiento de los bosques tropicales en el sureste del país, así como una de las más apreciadas en la industria del aserrío (Synnott, 2009; SEMARNAT, 2014). S. macrophylla es una de las especies más valiosas en el trópico y está sujeta a las normas internacionales del CITES (Convención sobre el Comercio Internacional de Especies Amenazadas de Fauna y Flora Silvestres; CONABIO, 2017). S. macrophylla King (Meliaceae) es una especie nativa del sureste de México, Centroamérica y el norte de Sudamérica (Brasil) (Rojas \& Torres, 2008). Los árboles de esta especie alcanzan dimensiones en promedio de $35 \mathrm{~m}$ de altura (Schmidt \& Jøker, 2000); pero existen reportes de diámetros con dimensiones de 1.5, 2 y $3.5 \mathrm{~m}$ (ESNACIFOR-OIMT, 2000; Vester \& Navarro-Martínez, 2007; Pérez, 2017). La densidad media oscila entre 0.3 a 1.9 ind/ha mayores a $55 \mathrm{~cm}$ de diámetro normal (Synnott, 2009; CONAFOR, 2014). Es una especie con un alto potencial para el establecimiento de 
plantaciones forestales en los trópicos, pero con un fuerte problema de barrenador de la yema terminal en plantas jóvenes (Montero, Valdez, De los Santos, Cetina, \& Sánchez, 2011; Marín et al., 2012; Pérez, DomínguezDomínguez, Martínez-Zurimendi, \& Etchevers Barra, 2012). Quintana Roo es uno de los estados con mayor aprovechamiento en este tipo de bosques en México (51 $947 \mathrm{~m}^{3}$ rollo), con el segundo lugar en extracción de madera preciosa (4 $807 \mathrm{~m}^{3}$ rollo) (SEMARNAT, 2016).

Debido a la importancia de estimar la edad y calcular el incremento en el manejo y aprovechamiento de los bosques tropicales (Del Valle, 1986, 1997; López-Torres \& Tamarit-Urias, 2005; Domínguez-Calleros et al., 2017). Los objetivos del presente trabajo fueron i) estimar la edad $\left(E_{t}\right)$ a partir del método del 'tiempo de paso' (Tp) y ii) calcular las tasas de crecimiento absoluto (TCA) y relativo (TCR) para S. macrophylla en los bosques tropicales de Felipe Carrillo Puerto, Quintana Roo, México. Hipotetizamos que es posible obtener una edad para esta especie al usar mediciones periódicas del diámetro normal.

\section{MATERIALES Y MÉTODOS}

El área de estudio: se localiza en el municipio de Felipe Carrillo Puerto Quintana Roo, México, en las regiones fisiográficas Carso Yucateco y Costa Baja de Quintana Roo con un sistema de topoformas características de la llanura rocosa (LRO) y Región Hidrológica Yucatán Este (RH33). El clima es cálido subhúmedo (Aw) con régimen de lluvias en verano con precipitación media anual de 1300 $\mathrm{mm}$ y temperatura que oscila entre los 24.6 ${ }^{\circ} \mathrm{C}$ y $28.4{ }^{\circ} \mathrm{C}$ donde los meses más secos son febrero, marzo y abril $(<17 \%$ de humedad $)$ (INEGI, 2016). La geología es de la era Cenozoica, periodo terciario, roca sedimentaria de litología caliza y suelos generalmente de tipo Litosol (L) y Regosol (R) (SEMARNAT, 2014; INEGI, 2016).

Las características de la vegetación son de bosque tropical perennifolio (15-30 $\mathrm{m}$ de altura) cuyas especies dominantes, ordenadas por su abundancia, son: Brosimum alicastrum Swartz. (Moraceae: Ramón), Cedrela odorata L. (Meliaceae: Cedro rojo), Bucida buceras L. (Combretaceae: Pucté), Manilkara zapota (L.) P. Royen (Sapotaceae: Chicozapote), Swietenia macrophylla King (Meliaceae: Caoba), Lonchocarpus castilloi Standl (Fabaceae: Machiche), Lysiloma latisiliquum (L.) Benth. (Fabaceae: Tzalam), Spondian mombin L. (Anacardiaceae: Jobo) y Bursera simaru$b a$ (L.) Sarg. (Burseraceae: Chakáh); además en menor abundancia Byrsonima bucidaefolia Standl. (Malpighiaceae: Sakpaj), Caesalpinia gaumeri (Britton, \& Rose) Greenm. (Fabaceae: Kitamché), Metopium brownei (Jacq.) Urb. (Anacardiaceae: Chechem negro) y Coccoloba acapulcensis Standl. (Polygonaceae: Xtojyuub) (CONABIO, 2008; Carreón-Santos \& ValdezHernández, 2014; SEMARNAT, 2014).

Se seleccionaron 403 árboles dominantes verticalmente por su posición social dentro del rodal de $S$. macrophylla, sin malformaciones o daños estructurales causados por vientos o huracanes y sin plagas y/o enfermedades visibles. En estos individuos se instaló, a una altura de $1.3 \mathrm{~m}$ sobre el fuste, una banda dendrométrica de aluminio graduada en milímetros para registrar el diámetro normal $(d)$ en períodos de $1,2,5,9,11$ y 15 años, después de medir el $d$ inicial $\left(d_{i}\right)$. Se cuantificó el incremento en $\mathrm{cm}$ para cada periodo de tiempo $(t)$, con la finalidad de calcular la tasa de crecimiento absoluto (TCA en $\mathrm{cm}^{2}$ año $^{-1}$ ) que representa el cambio en diámetro por unidad de tiempo y resulta de la diferencia entre el diámetro normal final $\left(d_{f}\right)$ y $d_{i}$, dividida entre la diferencia del tiempo final $\left(t_{f}\right)$ e inicial $\left(t_{i}\right)$ [1]. También se calculó la tasa de crecimiento relativo ( $T C R$ en $\mathrm{cm}$ año ${ }^{-1}$ ), la cual es una medida estandarizada de la capacidad productiva de un organismo y se obtiene al dividir la $T C A$ entre el $d_{i}$ [2] (Larocque \& Marshall, 1993; Pommerening \& Muszta, 2016). Posterior, se definió el tiempo de paso ( $T p$; Klepac, 1983; López-Torres \& Tamarit-Urias, 2005; Calvo-Rodríguez, Do Espírito-Santo, Nunes, \& Calvo-Alvarado, 2015; Canizales \& Aguirre, 2018). 


$$
\begin{gathered}
T C A=\frac{\left(d_{f}-d_{i}\right)}{\left(t_{f}-t_{i}\right)} \\
T C R=\frac{\left(d_{f}-d_{i}\right)}{\left(t_{f}-t_{i}\right)} / d_{i}=T C A / d_{i}
\end{gathered}
$$

Se eliminaron los puntos que no parecen corresponderse con el resto de los valores en el grupo de datos (puntos aberrantes) y los errores de captura al construir la base de datos. Para realizar el ajuste estadístico de las ecuaciones diferenciales que estiman la TCA más eficiente, se agrupó la información por categoría diamétrica $(C D)$ en intervalos de $5 \mathrm{~cm}$ (Del Valle, 1986, 1997; Manzano-Méndez et al., 2010; Lozano, Nathaly, Jeimy, \& Bonilla, 2012;

TABLA 1

Ecuaciones diferenciales ajustadas para calcular la tasa de crecimiento absoluto $(T C A)$ en diámetro para árboles de S. macrophylla en Quintana Roo, México

TABLE 1

Adjusted differential equations to calculate the absolute growth rate $(T C A)$ in diameter

\begin{tabular}{|c|c|c|}
\hline Número & Nombre & Ecuación \\
\hline [3] & Logística & $\frac{\vartheta(d)}{\vartheta t}=a \cdot d \cdot\left(1-\frac{d}{b}\right)+\epsilon$ \\
\hline$[4]$ & Logística-A & $\frac{\vartheta(d)}{\vartheta t}=a \cdot d \cdot\left(1-\frac{d}{180}\right)+\epsilon$ \\
\hline$[5]$ & Gompertz & $\frac{\vartheta(d)}{\vartheta t}=a \cdot d \cdot \log \left(\frac{d}{b}\right)+\epsilon$ \\
\hline$[6]$ & Von Bertalanffy & $\frac{\vartheta(d)}{\vartheta t}=a \cdot d^{b}-180^{b-1} \cdot d+\epsilon$ \\
\hline$[7]$ & Log. Normal & $\frac{\vartheta(d)}{\vartheta t}=a \cdot e^{-0.5 \cdot \frac{\left(\log \left(\frac{d}{c}\right)\right)^{2}}{b}}+\epsilon$ \\
\hline [8] & Chapman-Richards & 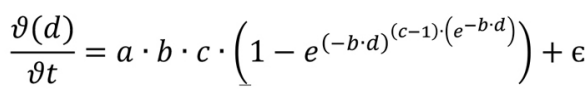 \\
\hline [9] & Chapman-Richards-A & 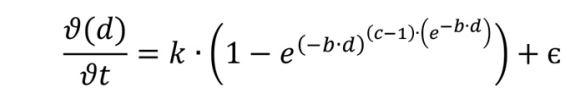 \\
\hline$[10]$ & Schumacher & $\frac{\vartheta(d)}{\vartheta t}=a \cdot b \cdot e^{-\frac{\frac{b}{d}}{d^{2}}}+\epsilon$ \\
\hline$[11]$ & Verhulst-Logística & $\frac{\vartheta(d)}{\vartheta t}=\frac{a \cdot c \cdot e^{b-c \cdot t}}{\left(1+e^{b-c \cdot t}\right)^{2}}+\epsilon$ \\
\hline$[12]$ & Hossfeld IV & $\frac{\vartheta(d)}{\vartheta t}=\frac{a \cdot c \cdot d^{c-1}}{\left(a+b \cdot d^{c}\right)^{2}}+\epsilon$ \\
\hline
\end{tabular}
for S. macrophylla trees in Quintana Roo, Mexico

$d$ : Diámetro normal (cm); $\vartheta d$ : Diferencial de incremento en diámetro $(\mathrm{cm})$; $\vartheta t$ : Diferencial de tiempo; $a, b, c$ y $k$ : Parámetros a estimar; $e$ : Base de logaritmos neperianos; $\mathrm{C}$ : Error.

$d$ : Normal diameter $(\mathrm{cm})$; $\vartheta d$ : Incremental differential in diameter $(\mathrm{cm})$; $\vartheta t$ : Time differential; $a, b, c$ and $k$ : Parameters to estimate; e: Base of Neperian logarithms; $\mathrm{C}$ : Error. 
TABLA 2

Ecuaciones para calcular la tasa de crecimiento relativo (TCR) en diámetro y el tiempo de paso (Tp) para árboles de S. macrophylla en Quintana Roo, México

TABLE 2

Equations to calculate the relative growth rate $(T C R)$ in diameter and the passage time $(T p)$ for S. macrophylla trees in Quintana Roo, Mexico

\begin{tabular}{clc} 
Número & \multicolumn{1}{c}{ Nombre } & Ecuación \\
{$[13]$} & Alométrica & $y=a \cdot d^{b}+\epsilon$ \\
{$[14]$} & Potencial & $y=a \cdot d^{b} \cdot c^{d}+\epsilon$ \\
{$[15]$} & Polinomial 2 ${ }^{\text {do }}$ orden & $y=a+b \cdot d+c \cdot d^{2}+\epsilon$ \\
{$[16]$} & Polinomial 3 ${ }^{\text {er }}$ orden & $y=a+b \cdot d+c \cdot d^{3}+\epsilon$ \\
\hline
\end{tabular}

d: Diámetro normal $(\mathrm{cm}) ; y$ : Tasa de crecimiento relativo $(T C R)$, Tiempo de paso $(T p) ; a, b$ y $c$ : Parámetros a estimar; C: error.

$d$ : Normal diameter $(\mathrm{cm}) ; y$ : Relative growth rate $(T C R)$, Step time $(T p) ; a, b$ and $c$ : Parameters to estimate; $\mathrm{E}$ : error.

Pineda-Herrera, Valdez-Hernández, PérezOlvera, \& Dávalos-Sotelo, 2015b). Luego con el procedimiento Model y la técnica de máxima verosimilitud ( $\mathrm{fiml}$ ), en el programa SAS 9.2 (SAS Institute Inc., 2014), se ajustaron diez ecuaciones diferenciales propuestas y utilizadas por Kiviste, Álvarez, Rojo y Ruiz (2002), Paine et al. (2012), Pineda-Herrera et al. (2015a, 2015b) y Domínguez-Calleros et al. (2017) para calcular la TCA (Tabla 1). Con el mismo procedimiento estadístico, ahora para calcular la $T C R$ y el $T p$, se ajustaron cuatro ecuaciones que han mostrado efectividad para modelar las tendencias en este tipo de información (Tabla 2; Meza, Mora, Chavés, \& Fonseca, 2003; Makocki, Valdez-Hernández \& García, 2012).

Para seleccionar la mejor ecuación, se realizó un análisis comparativo entre los valores resultantes del ajuste donde se tomó como referencia el menor valor de la raíz del cuadrado medio del error (RCME) y el mayor valor del coeficiente de determinación ajustado $\left(\mathrm{R}^{2}\right.$ ajus. (Pompa-García, De los Santos-Posadas, Zepeda-Bautista, \& Corral-Rivas, 2011; Vibrans, Moser, Zimermann, \& Mazaneuro, 2015). Posterior a ello, se verificó el cumplimiento de los supuestos de normalidad de los datos, homocedasticidad de los residuos y auto-correlación de los errores con las pruebas de Shapiro-Wilk
$(S W)$, White's $(W)$ y Breusch-Pagan (BP), así como Durbin-Watson $(D W)$, respectivamente.

La evaluación de la precisión en las estimaciones realizadas con las mejores ecuaciones seleccionadas, se realizó a través del sesgo $(E)$ y la diferencia agregada en porcentaje $(D A$ \%) (Prodan et al. 1997; Lencinas \& Mohr-Bell 2007). Estos indicadores estadísticos de precisión expresan las desviaciones de las predicciones con respecto a los datos reales al usar las ecuaciones, en la escala de la variable dependiente y de forma porcentual para la muestra, respectivamente. Una vez definidas las mejores ecuaciones por el menor valor en la RCME, el mayor valor en la $\mathrm{R}_{\text {ajus. }}^{2}$ y el cumplimiento de los supuestos de regresión para calcular la $T C A, T C R$ y tendencia del $T p$, se estimó la edad $\left(E_{t}\right)$ al acumular el $T p$ por $C D$ (Klepac, 1983; López-Torres \& Tamarit-Urias, 2005) y el incremento corriente anual (ICA: 17) mediante el cociente de las diferencias entre categorías $\mathrm{y}$ edades. Mientras que, el incremento medio anual (IMA: 18), se calculó con el cociente del $d$ entre la edad (Meza et al., 2003).

$$
\begin{gathered}
I C A=\left(C D_{2}-C D_{1}\right) /\left(t_{2}-t_{1}\right) \\
(I M A=d / t)
\end{gathered}
$$




\section{RESULTADOS}

Los estadísticos descriptivos indican que la agrupación por categorías diamétricas en intervalos de $5 \mathrm{~cm}$ fue adecuada para disminuir la amplitud y corregir la normalidad de los datos, ya que la media solo tuvo una diferencia de $1.08 \mathrm{~cm}$ en el diámetro y de $0.22 \mathrm{~cm}$ en su $T C A$, con respecto a los datos completos (Tabla $3)$. Con esta agrupación, los valores en desviación estándar y varianza de la muestra aumentaron para el diámetro, pero disminuyeron en la variable de interés $(T C A)$, así como el índice de curtosis y el coeficiente de asimetría que indicaron una distribución asimétrica positiva con forma leptocúrtica para el diámetro y negativa platicúrtica para la $T C A$.

No obstante que las diez ecuaciones diferenciales ajustadas tuvieron todos sus parámetros estadísticamente significativos (alfa $=$ 0.05 y $P r=0.0001)$, Verhulst-Logística [11] fue la que describió hasta el $87.5 \%$ de la variabilidad en los datos y presentó los valores más bajos en la $R C M E$ (Tabla 4). Al verificar los supuestos de regresión, la ecuación [4] no cumplió con el de normalidad, las expresiones

TABLA 3

Estadísticos descriptivos para 403 árboles de S. macrophylla en Quintana Roo, México

TABLE 3

Descriptive statistics for 403 S. macrophylla trees in Quintana Roo, Mexico

\begin{tabular}{lcccc}
\multicolumn{1}{c}{ Estadístico } & $d$ & \multicolumn{2}{c}{$T C A$} & $T C A$ \\
Número de observaciones & \multicolumn{2}{c}{ Datos observados } & Datos por $C D$ de $5 \mathrm{~cm}$ \\
Media & 3303 & 3303 & 48 & 48 \\
Mínimo & 42.15 & 0.69 & 43.23 & 0.47 \\
Máximo & 11.00 & 0.04 & 15.00 & 0.13 \\
Desviación estándar & 97.36 & 0.95 & 100.00 & 0.73 \\
Varianza de la muestra & 12.68 & 0.79 & 22.73 & 0.18 \\
Índice de curtosis & 160.64 & 0.63 & 516.48 & 0.03 \\
Coeficiente de asimetría & -0.32 & 20.03 & 0.12 & -1.22 \\
Nivel de confianza (95.0\%) & 0.01 & 3.98 & 0.79 & -0.25 \\
\hline
\end{tabular}

$d$ : Diámetro normal $(\mathrm{cm}) ; T C A$ : Tasa de crecimiento absoluto; $C D$ : Categoría diamétrica $(\mathrm{cm})$.

$d$ : Normal diameter $(\mathrm{cm}) ; T C A$ : Absolute growth rate; $C D$ : Diameter category $(\mathrm{cm})$.

TABLA 4

Resumen estadístico y valor de los parámetros estimados de las ecuaciones diferenciales ajustadas a la tasa de crecimiento absoluto (TCA) en diámetro para árboles de S. macrophylla en Quintana, Roo, México

TABLE 4

Statistical summary and value of the estimated parameters of the differential equations adjusted to the absolute growth rate (TCA) in diameter for S. macrophylla trees in Quintana, Roo, Mexico

\begin{tabular}{ccccccccc} 
Ecuación & $S C E$ & $R C M E$ & $R^{2}{ }_{\text {ajus. }}$ & Parámetro & Estimación & Eea & Valor $t$ & $\operatorname{Pr}>|t|$ \\
{$[3]$} & 0.2940 & 0.0799 & 0.8043 & $\mathrm{a}$ & 0.02184 & 0.0007 & 32.34 & $<0.0001$ \\
& & & & $\mathrm{~b}$ & 111.35380 & 2.2514 & 49.46 & $<0.0001$ \\
{$[4]$} & 1.1218 & 0.1545 & 0.2692 & $\mathrm{a}$ & 0.01502 & 0.0005 & 27.95 & $<0.0001$ \\
{$[5]$} & 0.6915 & 0.1226 & 0.5397 & $\mathrm{a}$ & -0.01014 & 0.0008 & -13.41 & $<0.0001$ \\
& & & & $\mathrm{~b}$ & 147.46900 & 8.3926 & 17.57 & $<0.0001$ \\
{$[6]$} & 0.6977 & 0.1232 & 0.5356 & $\mathrm{a}$ & 0.99798 & 0.0008 & 1328.55 & $<0.0001$ \\
& & & & $\mathrm{~b}$ & 0.98945 & 0.0008 & 1183.28 & $<0.0001$ \\
\hline
\end{tabular}


TABLA 4 (Continuación) / TABLE 4 (Continued)

\begin{tabular}{|c|c|c|c|c|c|c|c|c|}
\hline Ecuación & $S C E$ & $R C M E$ & $R_{a j u s .}^{2}$ & Parámetro & Estimación & Eea & Valor $t$ & $\operatorname{Pr}>|t|$ \\
\hline \multirow[t]{3}{*}{ [7] } & 0.3466 & 0.0878 & 0.7642 & $\mathrm{a}$ & 0.63845 & 0.0216 & 29.53 & $<0.0001$ \\
\hline & & & & $\mathrm{b}$ & -0.64526 & 0.0346 & -18.64 & $<0.0001$ \\
\hline & & & & $\mathrm{c}$ & 47.72433 & 1.2504 & 38.17 & $<0.0001$ \\
\hline \multirow[t]{3}{*}[8]{} & 0.2772 & 0.0785 & 0.8114 & $\mathrm{a}$ & 53.46822 & 2.2385 & 23.89 & $<0.0001$ \\
\hline & & & & $\mathrm{b}$ & 0.02878 & 0.0019 & 15.55 & $<0.0001$ \\
\hline & & & & $\mathrm{c}$ & 4.14895 & 0.3633 & 11.42 & $<0.0001$ \\
\hline \multirow[t]{3}{*}{ [9] } & 0.2772 & 0.0785 & 0.8114 & $\mathrm{k}$ & 6.38697 & 0.7730 & 8.26 & $<0.0001$ \\
\hline & & & & $\mathrm{b}$ & 0.02879 & 0.0019 & 15.56 & $<0.0001$ \\
\hline & & & & $\mathrm{c}$ & 4.15019 & 0.3633 & 11.42 & $<0.0001$ \\
\hline \multirow[t]{2}{*}[10]{} & 0.4711 & 0.1012 & 0.6864 & $\mathrm{a}$ & 104.45890 & 4.6130 & 22.64 & $<0.0001$ \\
\hline & & & & $\mathrm{b}$ & 90.82308 & 3.2131 & 28.27 & $<0.0001$ \\
\hline \multirow[t]{3}{*}{ [11] } & 0.1833 & 0.0638 & 0.8753 & a & 32.07179 & 1.2355 & 25.96 & $<0.0001$ \\
\hline & & & & b & 2.32962 & 0.1006 & 23.16 & $<0.0001$ \\
\hline & & & & $\mathrm{c}$ & 0.04252 & 0.0021 & 20.75 & $<0.0001$ \\
\hline \multirow[t]{3}{*}{ [12] } & 0.2469 & 0.0741 & 0.8320 & $\mathrm{a}$ & 1737.65400 & 788.00 & 2.21 & 0.0326 \\
\hline & & & & $\mathrm{b}$ & 0.01789 & 0.0008 & 22.53 & $<0.0001$ \\
\hline & & & & $\mathrm{c}$ & 2.73488 & 0.1142 & 23.94 & $<0.0001$ \\
\hline
\end{tabular}

$S C E$ : Suma de cuadrados del error; $R C M E$ : Raíz del cuadrado medio del error; $R_{\text {ajus }}^{2}$ Coeficiente de determinación ajustado; Eea: Error estándar aproximado; $a, b, c$ y $k$ : Parámetros estimados.

$S C E$ : Sum of error squares; $R C M E$ : Root of the mean square of the error; $R^{2}{ }_{\text {adjustment }}$ : Adjusted coefficient of determination; Eea: Approximate Standard Error; $a, b, c$ and $k$ : Estimated parameters.

[5] a [10] y [12] no cumplieron con el supuesto de una distribución homogénea de los residuos y las ecuaciones [3], [5] y [6] no satisficieron el de auto-correlación de los errores. Por lo cual, Verhulst-Logística [11] se confirma como la mejor ecuación para calcular la $T C A$ en árboles de S. macrophylla (Tabla 5). El sesgo individual y la diferencia agregada tuvieron sus valores más altos en la ecuación [5] y los más bajos en la ecuación seleccionada [11] (Fig. 1A, Fig. 1B).

Se observa que la ecuación Verhulst-Logística [11] fue la que presentó una mejor tendencia de la $T C A$ por categoría diamétrica (Fig. 2).

TABLA 5

Pruebas para verificar los supuestos de regresión en ecuaciones diferenciales ajustadas a la tasa de crecimiento absoluto (TCA) en diámetro para árboles de S. macrophylla en Quintana, Roo, México

TABLE 5

Tests to verify the regression assumptions in differential equations adjusted to the absolute growth rate $(T C A)$ in diameter for S. macrophylla trees in Quintana, Roo, Mexico

\begin{tabular}{|c|c|c|c|c|c|c|c|}
\hline \multirow{2}{*}{ Ecuación } & \multicolumn{2}{|c|}{ Normalidad } & \multicolumn{3}{|c|}{ Homocedasticidad } & \multicolumn{2}{|c|}{ Auto-correlación } \\
\hline & Prueba & Valor & Prueba & Valor & $P r>C h i^{2}$ & Prueba & Valor \\
\hline \multirow[t]{2}{*}[3]{} & SW & 0.97 & W & 1.41 & 0.8431 & DW & 1.16 \\
\hline & & & $\mathrm{BP}$ & 1.64 & 0.4402 & & \\
\hline \multirow[t]{2}{*}[4]{} & $S W$ & 0.75 & W & 13.87 & 0.0010 & DW & 1.50 \\
\hline & & & $\mathrm{BP}$ & 34.74 & $<0.0001$ & & \\
\hline \multirow[t]{2}{*}[5]{} & $S W$ & 0.96 & W & 18.95 & 0.0020 & DW & 1.00 \\
\hline & & & $\mathrm{BP}$ & 15.96 & 0.0003 & & \\
\hline \multirow[t]{2}{*}[6]{} & $S W$ & 0.96 & W & 19.41 & 0.0016 & DW & 1.00 \\
\hline & & & $\mathrm{BP}$ & 16.32 & 0.0003 & & \\
\hline
\end{tabular}


TABLA 5 (Continuación) / TABLE 5 (Continued)

\begin{tabular}{|c|c|c|c|c|c|c|c|}
\hline \multirow{2}{*}{ Ecuación } & \multicolumn{2}{|c|}{ Normalidad } & \multicolumn{3}{|c|}{ Homocedasticidad } & \multicolumn{2}{|c|}{ Auto-correlación } \\
\hline & Prueba & Valor & Prueba & Valor & $\operatorname{Pr}>C h i^{2}$ & Prueba & Valor \\
\hline \multirow[t]{2}{*}{ [7] } & $S W$ & 0.95 & W & 16.04 & 0.0248 & DW & 1.85 \\
\hline & & & $\mathrm{BP}$ & 15.43 & 0.0004 & & \\
\hline \multirow[t]{2}{*}[8]{} & $S W$ & 0.96 & W & 24.85 & 0.0017 & DW & 1.80 \\
\hline & & & $\mathrm{BP}$ & 11.52 & 0.0031 & & \\
\hline \multirow[t]{2}{*}{ [9] } & $S W$ & 0.96 & W & 12.57 & 0.0833 & DW & 1.80 \\
\hline & & & $\mathrm{BP}$ & 11.49 & 0.0032 & & \\
\hline \multirow[t]{2}{*}[10]{} & $S W$ & 0.95 & W & 21.80 & 0.0006 & DW & 1.92 \\
\hline & & & $\mathrm{BP}$ & 21.46 & $<0.0001$ & & \\
\hline \multirow[t]{2}{*}[11]{} & $S W$ & 0.96 & W & 6.48 & 0.5938 & DW & 1.53 \\
\hline & & & $\mathrm{BP}$ & 0.93 & 0.6288 & & \\
\hline \multirow[t]{2}{*}[12]{} & $S W$ & 0.96 & W & 16.96 & 0.0305 & DW & 1.78 \\
\hline & & & $\mathrm{BP}$ & 9.78 & 0.0075 & & \\
\hline
\end{tabular}

$S W$ : Shapiro Wilk; W: White; $B P$ : Breusch Pagan; $D W$ : Durbin Watson. $S W$ : Shapiro Wilk; $W$ : White; BP: Breusch Pagan; $D W$ : Durbin Watson.
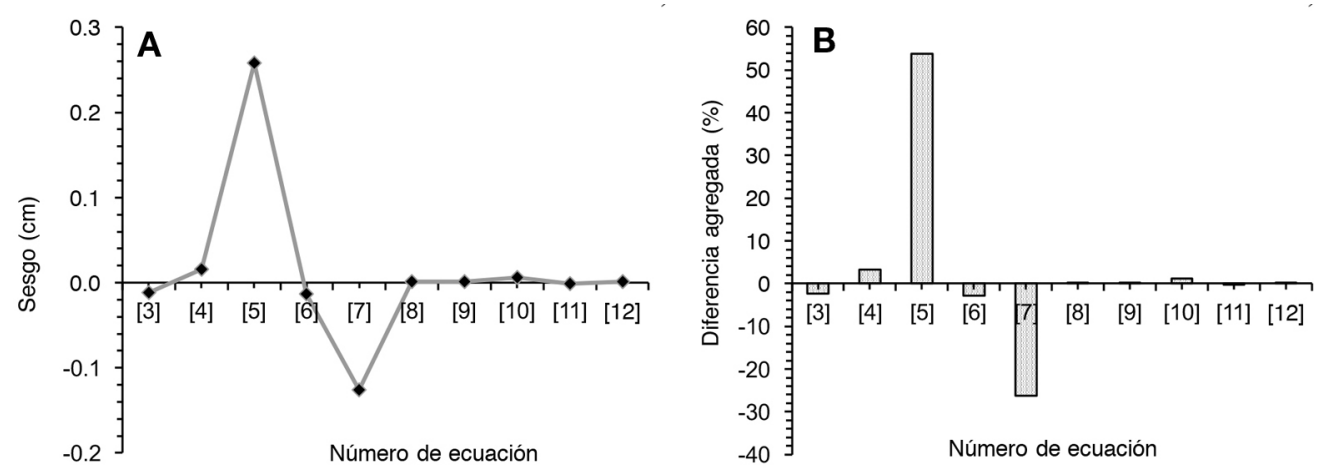

Fig. 1. Tasa de crecimiento absoluto (TCA) en diámetro para árboles de S. macrophylla en Quintana Roo, México, (A) Sesgo individual $(\mathrm{cm})$ y (B) diferencia agregada $(\%)$.

Fig. 1. Absolute growth rate (TCA) in diameter for S. macrophylla trees in Quintana Roo, Mexico, (A) Individual bias (cm) and (B) aggregate difference (\%).

Las ecuaciones [14] y [15] explicaron el $76 \%$ y el $82 \%$ de la variabilidad en la $T C R$ y en el $T p$, respectivamente, mostrando valores bajos en la $R C M E$ y parámetros estadísticamente significativos (Tabla 6). Sin embargo, al verificar los supuestos de la regresión, dichas ecuaciones no mostraron una distribución homogénea de los residuos ni ausencia de auto-correlación de los errores (Tabla 7).

Para cumplir con estos supuestos en las ecuaciones estadísticamente superiores en cada caso (14 y 15), se utilizó una regresión ponderada (Prodan et al., 1997; Álvarez-González, Rodríguez-Soalleiro, \& Rojo-Alboeca, 2007) con las variables $x^{2}, x^{3}, \operatorname{raiz}(x), \operatorname{raiz}\left(x^{2}\right)$, raiz $\left(x^{3}\right), 1 / x, 1 / x^{2}, 1 / x^{3}, 1 /$ raiz $(x), 1 /$ raiz $\left(x^{2}\right)$ y $1 /$ raiz $\left(x^{3}\right)$. A las cuales $1 /$ raiz $(x)$ para la ecuación [14] y $1 / x^{2}$ para la [15] fueron las variables que tuvieron los mejores ajustes, explicando más del $84 \%$ de la variabilidad en la TCR (ecuación [14.1], Tabla 6) y disminuyendo el valor de la $R C M E$ en el Tp (ecuación [15.1], Tabla 6). Con esta regresión ponderada se cumplieron los supuestos de distribución homogénea de los 


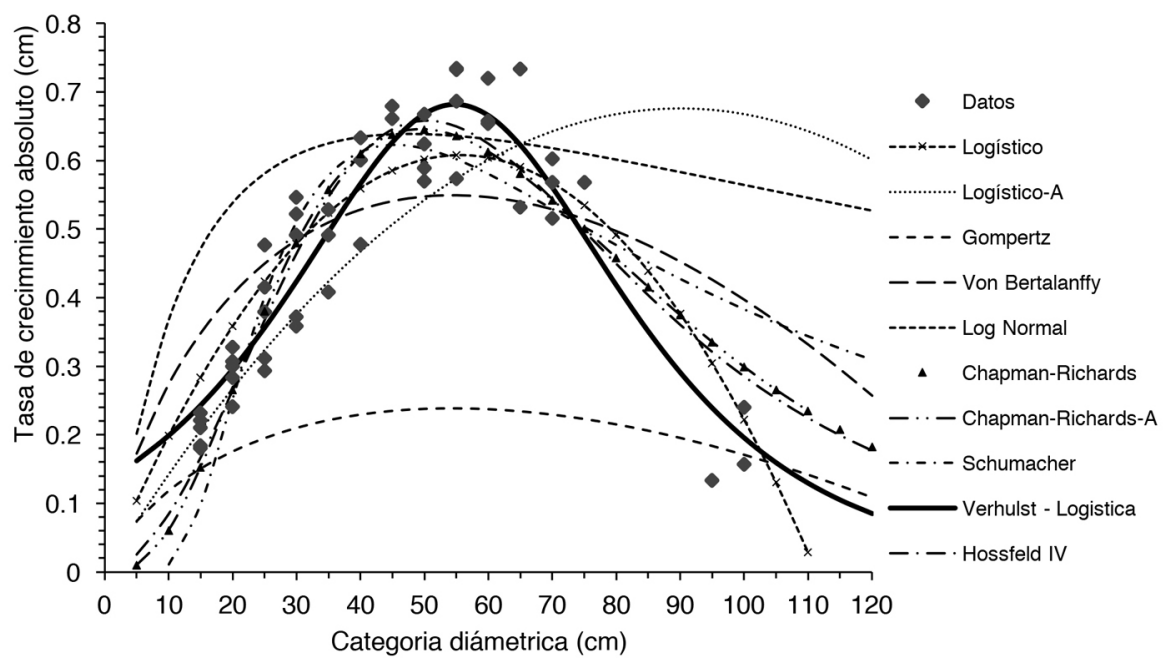

Fig. 2. Tendencia de la tasa de crecimiento absoluto $(T C A)$ por categoría diamétrica para árboles de $S$. macrophylla en Quintana Roo, México.

Fig. 2. Trend of absolute growth rate (TCA) by diameter category for S. macrophylla trees in Quintana Roo, Mexico.

TABLA 6

Resumen estadístico y parámetros estimados de las ecuaciones ajustadas a la tasa de crecimiento relativo (TCR) en diámetro y al tiempo de paso $(T p)$ por categoría diamétrica en árboles de $S$. macrophylla en Quintana Roo, México

TABLE 6

Statistical summary and estimated parameters of the equations adjusted to the relative growth rate $(T C R)$ in diameter and the passage time ( $T p$ ) by diametric category in $S$. macrophylla trees in Quintana Roo, Mexico

\begin{tabular}{|c|c|c|c|c|c|c|c|c|c|}
\hline $\begin{array}{c}\text { Variable } \\
\text { (y) }\end{array}$ & Ecuación & $S C E$ & $R C M E$ & $R_{a j u s}^{2}$ & Parámetro & Estimación & Eea & Valor $t$ & $\operatorname{Pr}>|t|$ \\
\hline \multirow[t]{14}{*}{ TCR } & [13] & 0.0004 & 0.0030 & 0.3863 & $\mathrm{a}$ & 0.040030 & 0.00769 & 5.21 & $<0.0001$ \\
\hline & & & & & $\mathrm{b}$ & -0.326380 & 0.05310 & -6.15 & $<0.0001$ \\
\hline & [14] & 0.0002 & 0.0019 & 0.7632 & $\mathrm{a}$ & 0.001281 & 0.00065 & 1.97 & 0.0545 \\
\hline & & & & & $\mathrm{b}$ & 1.070761 & 0.19700 & 5.44 & $<0.0001$ \\
\hline & & & & & $\mathrm{c}$ & 0.962407 & 0.00521 & 184.90 & $<0.0001$ \\
\hline & {$[15]$} & 0.0001 & 0.0018 & 0.7816 & $\mathrm{a}$ & 0.013636 & 0.00121 & 11.25 & $<0.0001$ \\
\hline & & & & & $\mathrm{b}$ & 0.000095 & 0.00006 & 1.68 & 0.1005 \\
\hline & & & & & $\mathrm{c}$ & $-2.24 \mathrm{E}-06$ & $5.3 \mathrm{E}-07$ & -4.18 & 0.0001 \\
\hline & [16] & 0.0002 & 0.0019 & 0.7675 & $\mathrm{~b}$ & -0.000020 & 0.00003 & -0.64 & 0.5253 \\
\hline & & & & & $\mathrm{c}$ & $-1.25 \mathrm{E}-08$ & $3.2 \mathrm{E}-09$ & -3.79 & 0.0004 \\
\hline & & & & & $\mathrm{a}$ & 0.015253 & 0.00092 & 16.55 & $<0.0001$ \\
\hline & [14.1] & 0.0016 & 0.0059 & 0.8455 & $\mathrm{a}$ & 0.000832 & 0.00040 & 2.06 & 0.0455 \\
\hline & & & & & $\mathrm{b}$ & 1.249148 & 0.18260 & 6.84 & $<0.0001$ \\
\hline & & & & & $\mathrm{c}$ & 0.957040 & 0.00424 & 225.90 & $<0.0001$ \\
\hline \multirow[t]{7}{*}{$\mathrm{Tp}$} & [13] & 517.40 & 3.3539 & 0.1380 & $\mathrm{a}$ & 37.311550 & 16.94320 & 2.20 & 0.0327 \\
\hline & & & & & $\mathrm{b}$ & -0.490510 & 0.11640 & -4.22 & 0.0001 \\
\hline & [14] & 124.00 & 1.6599 & 0.7889 & $\mathrm{a}$ & 8689.199000 & 5414.60 & 1.60 & 0.1155 \\
\hline & & & & & $\mathrm{b}$ & -2.751450 & 0.24650 & -11.16 & $<0.0001$ \\
\hline & & & & & $\mathrm{c}$ & 1.064702 & 0.00566 & 188.17 & $<0.0001$ \\
\hline & {$[15]$} & 104.40 & 1.5235 & 0.8222 & a & 18.551420 & 1.08810 & 17.05 & $<0.0001$ \\
\hline & & & & & $\mathrm{b}$ & -0.574510 & 0.05420 & -10.60 & $<0.0001$ \\
\hline
\end{tabular}


TABLA 6 (Continuación) / TABLE 6 (Continued)

\begin{tabular}{|c|c|c|c|c|c|c|c|c|c|}
\hline $\begin{array}{l}\text { Variable } \\
\text { (y) }\end{array}$ & Ecuación & $S C E$ & $R C M E$ & $R_{a j u s}^{2}$ & Parámetro & Estimación & Eea & Valor $t$ & $\operatorname{Pr}>|t|$ \\
\hline & \multirow{4}{*}{ [16] } & \multirow{3}{*}{121.80} & \multirow{3}{*}{1.6450} & \multirow{3}{*}{0.7927} & $\mathrm{c}$ & 0.005427 & 0.00046 & 11.94 & $<0.0001$ \\
\hline & & & & & $\mathrm{b}$ & -0.305870 & 0.03780 & -8.10 & $<0.0001$ \\
\hline & & & & & $\mathrm{c}$ & 0.000031 & 0.00000 & 10.06 & $<0.0001$ \\
\hline & & \multirow{4}{*}{1.25} & \multirow{4}{*}{0.1665} & \multirow{4}{*}{0.7879} & $\mathrm{a}$ & 14.947660 & 0.90280 & 16.56 & $<0.0001$ \\
\hline & \multirow[t]{3}{*}{ [15.1] } & & & & a & 15.836870 & 0.78610 & 20.15 & $<0.0001$ \\
\hline & & & & & $\mathrm{b}$ & -0.457620 & 0.03400 & -13.45 & $<0.0001$ \\
\hline & & & & & $\mathrm{c}$ & 0.004241 & 0.00032 & 13.07 & $<0.0001$ \\
\hline
\end{tabular}

$S C E$ : Suma de cuadrados del error. RCME: Raíz del cuadrado medio del error. $R_{\text {ajus }}^{2}$ : Coeficiente de determinación ajustado. Eea: Error estándar aproximado. $a, b$ y $c$ : Parámetros estadísticos estimados.

$S C E$ : Sum of squares of the error. RCME: Root of the mean square of the error. $R^{2}$ adjustment: Adjusted coefficient of determination. Eea: Approximate standard error. $a, b$ and $c$ : Estimated statistical parameters.

TABLA 7

Pruebas para verificar los supuestos de regresión para ecuaciones ajustadas a la tasa de crecimiento relativo (TCR) en diámetro y al tiempo de paso $(T p)$ por categoría diamétrica en árboles de $S$. macrophylla en Quintana Roo, México

TABLE 7

Tests to verify the regression assumptions for equations adjusted to the relative growth rate $(T C R)$ in diameter and the passage time (Tp) by diametric category in S. macrophylla trees in Quintana Roo, Mexico

\begin{tabular}{|c|c|c|c|c|c|c|c|c|}
\hline \multirow{2}{*}{ Variable $(y)$} & \multirow{2}{*}{ Ecuación } & \multicolumn{2}{|c|}{ Normalidad } & \multicolumn{3}{|c|}{ Homocedasticidad } & \multicolumn{2}{|c|}{ Auto-correlación } \\
\hline & & Prueba & Valor & Prueba & Estadístico & $\operatorname{Pr}>C h i^{2}$ & Prueba & Valor \\
\hline \multirow[t]{10}{*}{ TCR } & [13] & $S W$ & 0.96 & W & 35.44 & $<0.0001$ & $D W$ & 0.9 \\
\hline & & & & $\mathrm{BP}$ & 10.73 & 0.0047 & & \\
\hline & {$[14]$} & $S W$ & 0.95 & $W$ & 17.31 & 0.0155 & $D W$ & 1.2 \\
\hline & & & & $\mathrm{BP}$ & 2.76 & 0.2513 & & \\
\hline & {$[15]$} & $S W$ & 0.96 & $W$ & 11.61 & 0.0205 & $D W$ & 1.1 \\
\hline & & & & $\mathrm{BP}$ & 5.14 & 0.0765 & & \\
\hline & {$[16]$} & $S W$ & 0.96 & $W$ & 10.41 & 0.0643 & $D W$ & 1.1 \\
\hline & & & & $\mathrm{BP}$ & 5.1 & 0.0782 & & \\
\hline & {$[14.1]$} & $S W$ & 0.93 & $W$ & 18.31 & 0.0167 & $D W$ & 1.4 \\
\hline & & & & $\mathrm{BP}$ & 6.02 & 0.0494 & & \\
\hline \multirow[t]{10}{*}{$\mathrm{Tp}$} & [13] & $S W$ & 0.64 & $W$ & 30.66 & $<0.0001$ & $D W$ & 1.6 \\
\hline & & & & $\mathrm{BP}$ & 37.06 & $<0.0001$ & & \\
\hline & {$[14]$} & $S W$ & 0.93 & $W$ & 43.00 & $<0.0001$ & $D W$ & 1.9 \\
\hline & & & & $\mathrm{BP}$ & 25.96 & $<0.0001$ & & \\
\hline & {$[15]$} & $S W$ & 0.90 & $W$ & 24.93 & $<0.0001$ & $D W$ & 2.0 \\
\hline & & & & $\mathrm{BP}$ & 24.44 & $<0.0001$ & & \\
\hline & [16] & $S W$ & 0.90 & $W$ & 30.20 & $<0.0001$ & DW & 1.9 \\
\hline & & & & BP & 23.40 & $<0.0001$ & & \\
\hline & {$[15.1]$} & $S W$ & 0.99 & $W$ & 9.08 & 0.0592 & $D W$ & 1.6 \\
\hline & & & & $\mathrm{BP}$ & 8.28 & 0.0160 & & \\
\hline
\end{tabular}

$S W$ : Shapiro-Wilk; $W$ : White's; $B P$ : Breusch-Pagan; $D W$ : Durbin-Watson.

$S W$ : Shapiro Wilk; $W$ : White; BP: Breusch Pagan; $D W$ : Durbin Watson. 

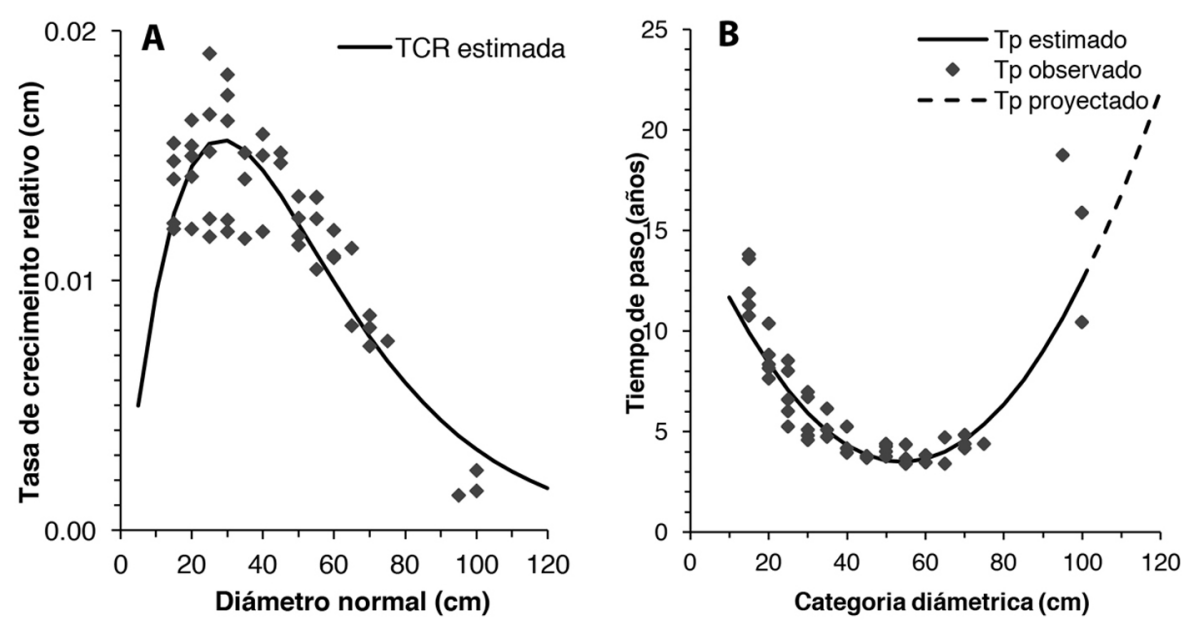

Fig. 3. Tasa de crecimiento relativo $(T C R)$ por categoría diamétrica para árboles de S. macrophylla en Quintana Roo, México: (A) En diámetro normal y (B) Por tiempo de paso (Tp).

Fig. 3. Relative growth rate $(T C R)$ by diametric category for S. macrophylla trees in Quintana Roo, Mexico: (A) In normal diameter and (B) By passage time $(T p)$.
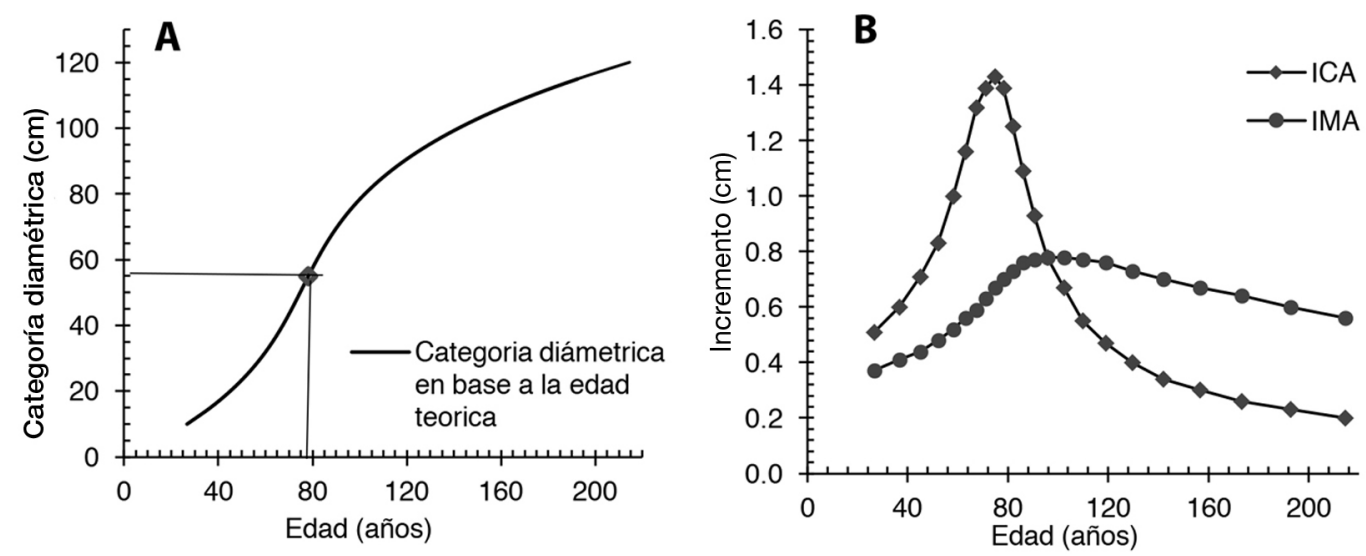

Fig. 4. Proyecciones para S. macrophylla en Quintana Roo, México, (A) Crecimiento en diámetro y (B) incrementos corriente (ICA) y medio (IMA) anuales.

Fig. 4. Projections for S. macrophylla in Quintana Roo, Mexico. (A) Annual growth in diameter and (B) average (ICA) and average (IMA) increments.

residuos y de no auto-correlación de los errores (Fuentes, Troncoso, \& Bonilla, 2001a, 2001b) para las ecuaciones [14.1: ] y [15.1: ] (Tabla 7).

Con las ecuaciones [14.1] (sesgo de $-0.00005 \mathrm{~cm}$; diferencia agregada $<1 \%)$ y [15.1] (sesgo de 0.23534 años; diferencia agregada $<1 \%$ ) se obtuvieron, respectivamente, las tendencias de la $T C R$ (Fig. 3A) y del $T p$ (Fig. 3B). Con la edad acumulada entre $C D$ se proyectó la edad a un diámetro normal especifico, tomando como referencia el diámetro mínimo de corta para bosques bajo manejo forestal en Quintana Roo establecido en $55 \mathrm{~cm}$, y el cual sucede aproximadamente a los 78 años (Fig. 4A). Una vez calculada la edad por $C D$ y con el uso de las ecuaciones [17] y [18], se proyectaron los incrementos corriente $(I C A)$ y medio (IMA) anuales, los cuales se intersectan 
TABLA 8

Edad acumulada e incrementos corriente $(I C A)$ y medio $(I M A)$ anuales por categoría diamétrica $(C D)$ para S. macrophylla en Quintana Roo México

TABLE 8

Cumulative age and current (ICA) and average (IMA) annual increases by diametric category $(C D)$ for S. macrophylla in Quintana Roo Mexico

\begin{tabular}{cccccccc} 
Edad (años) & $C D(\mathrm{~cm})$ & $I C A$ & $I M A$ & Edad (años) & $C D(\mathrm{~cm})$ & $I C A$ & $I M A$ \\
26.7 & 10 & 0.51 & 0.37 & 90.4 & 70 & 0.93 & 0.77 \\
36.6 & 15 & 0.60 & 0.41 & 95.8 & 75 & 0.78 & 0.78 \\
45.0 & 20 & 0.71 & 0.44 & 102.2 & 80 & 0.67 & 0.78 \\
52.0 & 25 & 0.83 & 0.48 & 109.7 & 85 & 0.55 & 0.77 \\
58.0 & 30 & 1.00 & 0.52 & 118.8 & 90 & 0.47 & 0.76 \\
63.0 & 35 & 1.16 & 0.56 & 129.4 & 95 & 0.40 & 0.73 \\
67.3 & 40 & 1.32 & 0.59 & 141.9 & 100 & 0.34 & 0.70 \\
71.1 & 45 & 1.39 & 0.63 & 156.4 & 105 & 0.30 & 0.67 \\
74.7 & 50 & 1.43 & 0.67 & 173.2 & 110 & 0.26 & 0.64 \\
78.2 & 55 & 1.39 & 0.70 & 192.5 & 115 & 0.23 & 0.60 \\
81.8 & 60 & 1.25 & 0.73 & 214.5 & 120 & 0.20 & 0.56 \\
85.8 & 65 & 1.09 & 0.76 & & & & \\
\hline
\end{tabular}

(turno técnico en diámetro) aproximadamente a una edad de 96 años (Fig. 4B).

Para facilitar la aplicación de la información en la elaboración de programas de manejo forestal para la especie, en la Tabla 8 se presenta la edad acumulada y los incrementos anuales (ICA, IMA) por CD para S. macrophylla en la región de Felipe Carrillo Puerto, Quintana Roo, México. Con base en lo anterior, el máximo $I C A$ ocurre a una edad aproximada de 75 años para un diámetro de $50 \mathrm{~cm}$, mientras que el máximo IMA y punto de cruce con el ICA se da alrededor de los 96 años para diámetros entre 75 y $80 \mathrm{~cm}$.

\section{DISCUSIÓN}

Los valores de asimetría y curtosis indicaron para el diámetro normal y la $T C A$ una distribución normal y adecuada de la muestra, ya que éstos fueron menores al doble del error estándar (-2 y 2) (Martínez, Sánchez, \& Faulin, 2006). Asimismo, el valor del coeficiente de determinación ajustado $\left(R_{\text {ajus }}^{2}=0.87\right)$ para la ecuación Verhulst-Logística en el cálculo de la $T C A$, se encuentra dentro de los intervalos aceptables reportados por Del Valle (1986) al ajustar ecuaciones diferenciales a distintas formas de agrupación en los diámetros; por Del Valle (1997) al comparar tres tipos de datos y establecer una asíntota desconocida y fija; y por Makocki et al. (2012) al utilizar ecuaciones polinomiales para modelar el incremento radial.

Verhulst-Logística es una ecuación diferencial de tipo exponencial decreciente que no pasa por el origen, pero sí presenta un punto de inflexión máximo (Kiviste et al., 2002). Además, asume que, en las etapas iniciales del individuo el crecimiento es exponencial hasta alcanzar un punto de inflexión y después inicia su disminución con un parámetro de autolimitación (Chiappa, Galindo, \& Cervantes, 2009). Esta tendencia es característica del crecimiento biológico de los organismos a medida que se hacen más longevos o la densidad por unidad de superficie aumenta (Bertalanffy, 1976; Chiappa et al., 2009; Gutiérrez \& Sánchez, 2017).

En S. macrophylla, la disminución de la velocidad de crecimiento en diámetros mayores a $55 \mathrm{~cm}$ se debe a la reducción del espacio de crecimiento y/o la disponibilidad de recursos, debido a que toda población está limitada por la capacidad de carga ambiental 
del lugar (Bertalanffy, 1976; Chiappa et al., 2009; Gutiérrez \& Sánchez, 2017) o el índice de sitio del área (García, Ramírez, Rodríguez, Jasso, \& Ortiz, 1998; García, Flores, \& Benavides, 2007; García, Rodríguez, \& Islas, 2011). Otro factor importante de auto-limitación es la longevidad del individuo, ya que desacelera la tasa de crecimiento hasta llegar a un punto que tiende a cero por los límites de recurso y espacio (Accinelli \& Brida, 2006; Bonilla \& Zaragoza, 2011).

El turno comercial en $S$. macrophylla es establecido por el diámetro mínimo de corta (DMC) mayor o igual que $55 \mathrm{~cm}$ (Santos, Carreón, \& Nelson, 1998; Reuter, Schulz, \& Marrufo, 1998; Synnott, 2009) y que de acuerdo a los resultados de este trabajo se alcanza a $\operatorname{los} 78$ años cuando se tiene un máximo en la $T C A\left(0.68 \mathrm{~cm}^{2}\right.$ ño $\left.^{-1}\right)$ y donde es el momento en el que el $T p$ empieza a aumentar. Esta tendencia, concuerda con la biología de una especie pionera longeva (Medina, Wang, Lugo, \& Popper, 2003; Van Rheenen, Boot, Werger, \& Ulloa, 2004) y demandante de luz que presenta un crecimiento acelerado, pero que puede establecerse bajo sombra (Grogan, Barreto, \& Veríssimo, 2002; Fetcher, Wen, Montaña, \& De Sastro, 2003; Medina et al., 2003). Si no se hace un adecuado manejo o se realiza una apertura de espacios de crecimiento en el tiempo exacto, S. macrophylla puede sobrevivir en condiciones de poca luminosidad, pero con incrementos muy pequeños o nulos.

Además, el $D M C$ es igual al definido por MIQROO (Maderas Industrializadas de Quintana Roo) desde 1954 (Barton \& Merino, 2004), y estipulado en la guía para el manejo de esta especie propuesta por la CONABIO (2017). De igual forma, la edad a la cual se alcanza el $D M C$, es menor a la reportada por Snook (2000) para bosques tropicales de Quintana Roo, México (86 años) esto posiblemente por la forma de cómo la autora obtuvo la edad del arbolado y calculó los incrementos para las condiciones de crecimiento propuestas. Sin embargo, la TCA es inferior a la reportada por Shono y Snook (2006) $\left(T C A=0.86 \mathrm{~cm}^{2}\right.$ año $^{-}$ $\left.{ }^{1}\right)$ para este mismo diámetro en los bosques naturales de Belice donde las condiciones del sitio presentan una mayor productividad que en la región evaluada.

La $T C A$ encontrada en este trabajo demuestra matemáticamente la velocidad de incremento para $S$. macrophylla y el punto en el cual las tasas de crecimiento llegan al nivel asintótico y comienzan a decrecer. Por lo cual, se difiere con lo propuesto por Cámara y Snook (1998), Snook (2003), Brown, Jennings, y Clements (2003) de incrementar el $D M C$ a dimensiones superiores de $75 \mathrm{~cm}$ en diámetro esto posiblemente debido a la elección de la muestra para el análisis, la forma de como definen la edad y al procedimiento matemático utilizado por estos autores. Para S. macrophylla, el turno técnico en diámetro se alcanza a los 96 años de edad $\left(0.78 \mathrm{~cm}\right.$ año $\left.{ }^{-1}\right)$. Esto difiere con el turno de 120 años reportado por Snook (2000) y Snook (2003) para rodales mixtos de $S$. macrophylla en Quintana Roo, México, aunque es semejante al turno técnico de 100 años mencionado por Santos et al. (1998) y Alcalá (2011) para las selvas de la zona de Felipe Carillo Puerto, Quintana Roo. De igual manera, los incrementos obtenidos $\left(0.78 \mathrm{~cm}\right.$ año $\left.{ }^{-1}\right)$ a esta dimensión, son superiores a los reportados por Alder (1997) en México (0.46 y $\left.0.39 \mathrm{~cm} \mathrm{año}^{-1}\right)$, pero inferiores a los mencionados por Shono y Snook (2006) $(0.86 \mathrm{~cm}$ y $1.38 \mathrm{~cm}$ año-1) para $S$. macrophylla en bosques naturales de Belice.

Debido a que se apoya la hipótesis y fue posible obtener una edad para esta especie al usar mediciones periódicas del diámetro normal en combinación el cumplimiento de los objetivos de este trabajo, se puede proponer para $S$. macrophylla un aclareo por lo bajo (método alemán) a los $28.5 \mathrm{~cm}$ de diámetro a una edad de 55 años, que es cuando se alcanza la máxima $T C R(0.0156) \mathrm{cm}^{2} \mathrm{ano}^{-1}$. La finalidad de proponer esta actividad silvícola es la de generar una apertura de espacios de crecimiento y acelerar el incremento en la especie tal y como lo documentan Gadow, Sánchez, y Aguirre (2004), Rojas (2006) y Leak (2007), al proponer un manejo forestal con bases científicas y registrar la respuesta de la masa forestal a los aclareos. 
Finalmente, ante la ausencia de anillos de crecimiento o de no conocer la edad de los árboles de $S$. macrophylla, a partir de mediciones periódicas del diámetro normal y el método de tiempo de paso es posible estimar las edades y calcular los crecimientos e incrementos en diámetro para Swietenia macrophylla de forma confiable. Además el valor máximo en la tasa de crecimiento absoluto permite definir el turno comercial para la especie a un diámetro mínimo de corta de $55 \mathrm{~cm}$ y una edad de 78 años, en tanto que el turno técnico se propone a los 96 años, mientras que la máxima tasa de crecimiento relativo indica que a $\operatorname{los} 28.5 \mathrm{~cm}$ y 55 años de edad, se deberá de aplicar un aclareo por lo bajo con la finalidad de controlar la densidad y propiciar nuevamente el incremento diamétrico en $S$. macrophylla en bosques naturales en el área de estudio.

Declaración de ética: los autores declaran que todos están de acuerdo con esta publicación y que han hecho aportes que justifican su autoría; que no hay conflicto de interés de ningún tipo; y que han cumplido con todos los requisitos y procedimientos éticos y legales pertinentes. Todas las fuentes de financiamiento se detallan plena y claramente en la sección de agradecimientos. El respectivo documento legal firmado se encuentra en los archivos de la revista.

\section{AGRADECIMIENTOS}

Al Instituto Nacional de Investigaciones Forestales, Agrícolas y Pecuarias (INIFAP) por el otorgamiento del apoyo a capacitación y formación de personal investigador, y al Consejo Nacional de Ciencia y Tecnología (CONACyT) por la beca número 733112 al primer autor.

\section{RESUMEN}

Introducción: Swietenia macrophylla King ("caoba") es la especie arbórea de mayor importancia comercial en el sureste mexicano y utilizada como guía en el manejo forestal, por lo que conocer su edad y tasas de crecimiento es fundamental para la sostenibilidad de los aprovechamientos maderables. Objetivos: Estimar la edad $\left(E_{t}\right)$ a partir del método del 'tiempo de paso' $(T p)$ y calcular las tasas de crecimiento absoluto (TCA) y relativo $(T C R)$ para Swietenia macrophylla en Quintana Roo, México. Métodos: Se ajustaron ecuaciones para calcular $T C A$ y $T C R$ con remediciones periódicas durante 15 años del diámetro normal $(d)$ en 403 árboles de $S$. macrophylla en Felipe Carrillo Puerto, Quintana Roo, México; también, se determinaron el $T p$ y la $E_{t}$ para el cálculo del incremento corriente anual (ICA) e incremento medio anual (IMA), y definir así los turnos comercial y técnico. Resultados: La máxima TCR se alcanzó a los $28 \mathrm{~cm}$ de $d\left(0.015 \mathrm{~cm}\right.$ año $\left.{ }^{-1}\right)$ (aplicación de aclareos), mientras que la mayor TCA (0.69 $\mathrm{cm}$ año $\left.{ }^{-1}\right)$ y menor $T p(3.5$ años) a los $55 \mathrm{~cm}$ de $d$ (turno comercial). El máximo $I C A$ al considerar la $E_{t}$ fue de 1.43 $\mathrm{cm}$ en la categoría diamétrica de $50 \mathrm{~cm}$, con un IMA de $0.67 \mathrm{~cm}$ año ${ }^{-1}$ a una edad de 75 años, mientras que el turno técnico $(I C A=I M A)$ se alcanzó a los 96 años (incrementos de $\left.0.78 \mathrm{~cm} \mathrm{año}^{-1}\right)$. Conclusiones: A través de mediciones periódicas del diámetro normal en $S$. macrophylla es posible obtener su edad, además los resultados pueden ser utilizados para elaborar programas de manejo y ejecutar prácticas silvícolas que propicien el incremento diamétrico en Quintana Roo.

Palabras clave: aprovechamiento maderable, caoba, tasas de crecimiento, tiempo de paso.

\section{REFERENCIAS}

Accinelli, E., \& Brida, J.G. (2006). Crecimiento económico óptimo y crecimiento poblacional: una versión mejorada del modelo de Ramsey. Papeles de Población, 12(47), 228-241.

Alcalá, M.R.E. (2011). Ecología, genética y conservación de la caoba (Swietenia macrophylla): herramientas para un manejo adaptativo de la selva Maya de Quintana Roo, México (Reporte técnico, SNIBCONABIO proyecto No. FQ006). México: Universidad Autónoma del Estado de Morelos-Centro de Investigación en Biodiversidad y Conservación.

Alder, D. (1997). Report on a consultancy to the Quintana Roo Forest Management Project. Oxford, United Kingdom: Denis Alder (Consulting) Ltd.

Álvarez-González, J.G., Rodríguez-Soalleiro, R., \& RojoAlboeca, A. (2007). Resolución de problemas de ajuste simultáneo de sistemas de ecuaciones: heterocedasticidad y variables dependientes con distinto número de observaciones. Cuadernos de la Sociedad Española de Ciencias Forestales, 23, 35-42.

Baker, T.R., Affum-Baffoe, K., Burslem, D.F.R.P., \& Swaine, M.D. (2002). Phenological differences in tree water use and the timing of tropical forest inventories: conclusions from patterns of dry season diameter change. Forest Ecology and Management, 171, 261-274. 
Barton, B.D., \& Merino, P.L. (2004). La experiencia de las comunidades forestales en México. Veinticinco años de silvicultura y construcción de empresas forestales comunitarias. México: INE-SEMARNAT.

Bertalanffy, L.V. (1976). Teoría de los sistemas: fundamentos, desarrollo y aplicaciones. México: Fondo de Cultura Económica.

Bonilla, G.J.A., \& Alarcón, N.J.A. (2015). Turnos técnico y económico de tala para arboles de romerillo blanco en Ecuador. Ecología Aplicada, 14(2), 127-137.

Bonilla, S.A., \& Zaragoza, B.J. (2011). La exploración intensiva y extensiva de la naturaleza. Ideas sobre su posible modelación matemática. Dimensión Económica, 2(6), 37-48.

Brown, N., Jennings, S., \& Clements, T. (2003). The ecology, silviculture and biogeography of mahogany (Swietenia macrophylla): a critical review of the evidence. Perspectives in Plant Ecology, Evolution and Systematics, 6, 37-49.

Calvo-Rodríguez, S., Do Espírito-Santo, M.M., Nunes, Y.R.F., \& Calvo-Alvarado, J. (2015). Tree diameter growth for three successional stages of tropical dry forest in Minas Gerais, Brazil. Revista Forestal Mesoamericana Kurú, 14(35), 24-32.

Cámara, L., \& Snook, L.K. (1998). Fruit and seed production by mahogany Swietenia macrophylla trees in the natural tropical forests of Quintana Roo, Mexico. TRI News, 17, 18-21.

Canizales, V.P.A., \& Aguirre, A.C. (2018). Determinación de la edad en especies maderables del trópico y subtrópico. Revista de Divulgación Científica y Tecnológica de la Universidad Autónoma de Nuevo León, 21(88), 1-1.

Carreón-Santos, R.J., \& Valdez-Hernández, J.I. (2014). Estructura y diversidad arbórea de vegetación secundaria derivada de una selva mediana subperennifolia en Quintana Roo. Revista Chapingo Serie Ciencias Forestales y del Ambiente, 20(1), 119-130.

Casals, C.V. (2005). El turno forestal, la propiedad de los montes y la recepción de la fórmula de Faustmann en España, 1849-1918. Revista Electrónica de Geografia y Ciencias Sociales, 9, 181-2014.

Chiappa, C.X., Galindo, S.M.C., \& Cervantes, S.A. (2009). Introducción a los modelos matemáticos de crecimiento con aplicación en sistemas biológicos. México: UNAM - UMDI - Sisal.

Clutter, J.L., Forston, J.C., Pienaar, L.V., Brister, G.H., \& Bailey, R.L. (1983). Timber management: A quantitative approach. New York, EE.UU.: John Wiley \& Sons Inc.

CONABIO [Comisión Nacional para la Biodiversidad] (2008). Capital natural de México, vol. I:
Conocimiento actual de la biodiversidad. México: Comisión Nacional para el Conocimiento y Uso de la Biodiversidad.

CONABIO (2017). Guía complementaria para la elaborar programas de manejo forestal sustentable y documentos técnicos unificados que incluyan aprovechamiento de caoba (Swietenia macrophylla King) en el marco de las disposiciones de la CITES. México: Comisión Nacional para el Conocimiento y Uso de la Biodiversidad.

CONAFOR [Comisión Nacional Forestal] (2014). Silvicultura de las selvas de caoba en Quintana Roo, México: criterios y recomendaciones. México: CONAFOR. $186 \mathrm{p}$.

Dykstra, D.P., \& Heinrich, R. (1996). Código modelo de prácticas de aprovechamiento forestal de la FAO. Italia: Organización de las Naciones Unidas para la Agricultura y la Alimentación.

Del Valle, A.J.I. (1986). La ecuación de crecimiento de von Bertalanffy en la determinación de la edad y el crecimiento de árboles tropicales. Revista Facultad Nacional de Agronomía, 39(1), 61-74.

Del Valle, A.J.I. (1997). Crecimiento de cuatro especies de los humedales forestales del litoral pacífico colombiano. Revista de la Academia Colombiana de Ciencias Exactas, Físicas y Naturales, 21(81), 445-466.

Domínguez-Calleros, P.A., Rodríguez-Flores, F.J., Lizárraga-Mendiola, L., Jiménez-Gómez, M.A., \& Navar, J. (2017). Aplicaciones y ejemplos de modelos de crecimiento diamétrico para árboles tropicales. Ecosistemas y Recursos Agropecuarios, 4(11), 265-274.

ESNACIFOR-OIMT [Escuela Nacional de Ciencias Forestales - Organización Internacional de las Maderas Tropicales] (2000). Caoba: Swietenia macrophylla G. King Colección Maderas Tropicales de HondurasPD 8/92 Rev. 2(F) (Ficha Técnica No. 25). Honduras: ESNACIFOR-OIMT.

Fetcher, N., Wen, S., Montaña, A., \& De Sastro F. (2003). Photosynthetic response of hybrid mahogany grown under contrasting light regimes. En A. Lugo, J.C. Figueroa Colón \& M. Alayón (Eds.), Big-leaf mahogany: Genetics, ecology and management (pp. 117128). EE.UU.: Springer.

Fuentes, E.D., Troncoso, J.J., \& Bonilla, C.A. (2001a). Operaciones forestales y concentración de sedimentos en cauces naturales I: Formulación de un modelo matemático. Bosque, 22, 15-22.

Fuentes, E.D., Troncoso, J.J., \& Bonilla, C.A. (2001b). Operaciones forestales y concentración de sedimentos en cauces naturales II: Análisis de sensibilidad y comparación con otros modelos. Bosque, 22, 25-27. 
Gadow, K.V., Sánchez, O.S., \& Aguirre, C.O.A. (2004) Manejo forestal con bases científicas. Madera y Bosques, 10(2), 3-16.

García, C.X., Ramírez, M.H., Rodríguez, F.C., Jasso, M.J., \& Ortiz, S.C.A. (1998). Índice de sitio para caoba (Swietenia macrophylla King) en Quintana Roo, México. Ciencia Forestal en México, 23(84), 9-18.

García, C.X., Flores, G.J., \& Benavides, S.J.D. (2007). Índice de sitio para Cedrela odorata L. (Cedro rojo) en Quintana Roo, México. Ciencia Forestal en México, 32(101), 71-92.

García, C.X., Rodríguez, S.B., \& Islas, G.J. (2011). Evaluación financiera de plantaciones forestales de caoba en Quintana Roo. Revista Mexicana de Ciencias Forestales, 2(7), 7-26.

Grogan, J., Barreto, P., \& Veríssimo, A. (2002). Mahogany in the Brazilian Amazon: Ecology and perspectives on management. Brazil: Imazon.

Gutiérrez, S.J.L., \& Sánchez, G.F. (2017). Matemática del crecimiento orgánico: De la alométria al crecimiento estacional. México: Editorial de la Universidad Nacional Autónoma de México.

Hart, P. (2010). Tree growth and age in an ancient Hawaiian wet forest: vegetation dynamics at two spatial scales. Journal of Tropical Ecology, 26, 1-11.

Hernández, L., \& Castellanos, H. (2006). Crecimiento diamétrico arbóreas en bosques de sierra de Lema, Guayana Venezolana: Primeras evaluaciones. Interciencia, 31(11), 779-786.

Imaña, E.J., \& Encinas, B.O. (2008). Epidometría Forestal. Brasil: Editorial Universidad de Brasilia-Departamento de Engenharia Florestal.

INEGI [Instituto Nacional de Estadística y Geografía] (2016). Anuario estadístico y geográfico de Quintana Roo 2016. México: INEGI. Recuperado de http:// internet.contenidos.inegi.org.mx/contenidos/Productos/prod_serv/contenidos/espanol/bvinegi/productos/ nueva estruc/anuarios 2016/702825084370.pdf

Interián-Ku, V.M., Vaquera-Huerta, H., Valdez-Hernández, J.I., García-Moya, E., \& Romero-Manzanares, A. (2014). Influencia de factores morfológicos y ambientales sobre el crecimiento en diámetro de Caesalpinia gaumeri Greenm en un bosque tropical caducifolio, en México. Revista Chapingo Serie Ciencias Forestales y del Ambiente, 20(3), 255-270.

Kiviste, A.J.G., Álvarez, G., Rojo, A.A., \& Ruiz, G.A.D. (2002). Funciones de crecimiento de aplicación en el ámbito forestal. España: Ministerio de Ciencia y Tecnología.

Klepac, D. (1983). Crecimiento e incremento de árboles y masas forestales (2 ${ }^{\text {da }}$ Ed.). México: Universidad Autónoma Chapingo.
Leak, W.B. (2007). Thinning northern hardwoods in New England by dominant tree removal early results. Northern Journal of Applied Forestry, 24, 312-313.

Lencinas, J.D., \& Mohr-Bell, D. (2007) Estimación de clases de edad de las plantaciones de la provincia de Corrientes, Argentina, con base en datos satelitales Landsat. Bosque, 28(2), 106-118.

Larocque, G.R., \& Marshall, P.L. (1993). Evaluating the impact of competition using relative growth rate in red pine (Pinus resinosa Ait.) stands. Forest Ecology and Management, 58, 65-83.

López-Ayala, J.L., Valdez-Hernández, J.I., Terrazas, T., \& Valdez-Lazalde, J.R. (2006a). Anillos de crecimiento y su periodicidad en tres especies tropicales del estado de Colima, México. Agrociencia, 40(4), 533-544.

López-Ayala, J.L., Valdez-Hernández, J.I., Terrazas, T., \& Valdez-Lazalde, J.R. (2006b). Crecimiento en diámetro de especies arbóreas en una selva mediana subcaducifolia en Colima, México. Agrociencia, 40(1), 139-147.

López-Hernández, M.I., Cerano-Paredes, J., ValenciaManzo, S., Cornejo-Oviedo, E.H., Villanueva-Díaz, J., Cervantes-Martínez, R., \& Esquivel-Arriaga, G. (2018). Respuesta del crecimiento de Pinus oocarpa a variables climáticas en Chiapas, México. Revista de Biología Tropical, 66(4), 1580-1596.

López-Torres, J.L., \& Tamarit-Urias, J.C. (2005). Crecimiento e incremento en diámetro de Lysiloma latisiliquum (L.) Benth. en bosques secundarios en Escárcega, Campeche, México. Revista Chapingo Serie Ciencias Forestales y del Ambiente, 11(2), 117-123.

Lozano, B.L.A., Nathaly, F.O., \& Bonilla, V.J.L. (2012). Estimación del crecimiento diamétrico, de Anacardium excelsum (Kunth) Skeels, por medio de modelos no lineales, en bosques naturales del departamento del Tolima. Boletín Científico, Centro de Museos, Museo de Historia Natural, 16(1), 19-32.

Manzano-Méndez, F., Valdez-Hernández, J.I., LópezLópez, M.A., \& Vaquera-Huerta, H. (2010). Crecimiento en diámetro de Zanthoxylum kellermanii $\mathrm{P}$. Wilson en una selva perennifolia del norte de Oaxaca, México. Madera y Bosques, 16(2), 19-33. DOI: 10.21829/myb.2010.1621170.

Makocki, M., Valdez-Hernández, J.I., \& García, M.E. (2012). Crecimiento de tres especies arbóreas en una selva mediana subcaducifolia de Nayarit. En P.E. Salcedo, A.E. Hernández, G.J.A. Vázquez, G.T. Escoto, \& E. N. Díaz (Eds.), Recursos Forestales en el Occidente de México (pp. 182-206). México: Amaya ediciones S. R. L.

Marín Hernández, T.D.N.J., Azpíroz Rivero, H.S., Parraguirre Lezama, C., Sánchez Monsalvo, V., Velasco Bautista, E., Hernández Juárez, R., Sánchez, M.A., \& 
Rueda Sánchez, A. (2012). Análisis de dimensiones dasométricas y porcentaje del ataque de Hysipyla grandella Zeller en Cedrela odorata L. Revista Mexicana de Ciencias Forestales, 3(13), 29-48.

Martínez, G.M.A., Sánchez, V.A., \& Faulin, F.J. (2006). Bioestadística amigable (2da Ed.) España: Ediciones Díaz de Santos.

Medina, E., Wang, H.H., Lugo, A.E., \& Popper, N. (2003). Growth-, water- and nutrient-related plasticity in hybrid mahogany leaf development under contrasting light regimes. En A. Lugo, J.C. Figueroa Colón, \& M. Alayón (Eds.), Big-leaf mahogany: Genetics, ecology and management (pp. 146-168). EE.UU.: Springer.

Meza, V., Mora, F., Chavés, E., \& Fonseca, W. (2003). Crecimiento y edad del bosque natural con y sin manejo en el trópico húmedo de Costa Rica. En XII Congreso Forestal Mundial. Quebec, Canadá. Recuperado de http://www.fao.org/3/XII/0063-B4.htm

Meza, M.A., \& Torres, C.G. (2006). El raleo: una operación silvicultural fundamental. Solución tecnológica. Revista Forestal Mesoamericana Kurú, 3(8), 1-3.

Montero, S.F.M., Valdez, H.J.I., De los Santos, P.H.M., Cetina, A.V.M., \& Sánchez, V.L.R. (2011). Crecimiento inicial de especies arbóreas multipropósito en un terreno ganadero del norte de Veracruz. Revista Mexicana de Ciencias Forestales, 2(3), 53-68.

Musálem, M.A. (1989). Los aclareos en plantaciones de árboles de uso múltiple. Definición, método y grado de los aclareos. En M.A. Musálem (Ed.), Memorias del I y II curso centroamericano de silvicultura de plantaciones de especies de árboles de usos múltiples. Recuperado de:

ht tps://books.google.com.mx/books? $\mathrm{id}=$ KcgOAQAAIAAJ\&pg $=$ PR8-IA68\&lpg=PR8IA $68 \& \mathrm{dq}=$ Aclareo + por $+10+$ bajo $\&$ source $=$ b1 $\&$ ots $=9$ uPobLFLTZ\&sig $=$ ACfU3U3z52XpPYFfva7K bp5kVR0oj49n9w\&hl=es\&sa=X\&ved=2ahUKEwi rg-Tp6YTiAhUPcq0KHWhIAec4ChDoATADegQ ICBAB $\# \mathrm{v}=$ onepage $\& \mathrm{q}=$ Aclareo $\% 20$ por $\% 2010 \% 20$ bajo $\& \mathrm{f}=$ false

Paine, C.E.T., Marthews, T.R., Vogt, D.R., Purves, D., Rees, M., Hector, A., \& Turnbull, L.A. (2012). How to fit nonlinear plant growth models and calculate growth rates: an update for ecologists. Methods in Ecology and Evolution, 3, 245-256. DOI: 10.1111/j.2041-210X.2011.00155.x.

Pérez, G.G., Domínguez-Domínguez, M., Martínez-Zurimendi, P., \& Etchevers, B.J.D. (2012). Caracterización dasométrica e índice de sitio en plantaciones de caoba en Tabasco, México. Madera y Bosques, 18(1), 7-24.

Pérez, V.J.M. (2017). Manual para el cultivo de la caoba. Serie Técnica No. 1. España: Instituto Laudato Si'.
Pineda-Herrera, E., Valdez-Hernández, J.I., \& LópezLópez, M.A. (2012). Fenología de Schizolobium parahyba y Vochysia guatemalensis en una selva alta perennifolia de Oaxaca, México. Botanical Sciences, 90(2), 185-193.

Pineda-Herrera, E., Valdez-Hernández, J.I., López-López, M.A., Manzano-Méndez, F., \& Salgado-Ugarte, I.H. (2015a). Incremento en diámetro y periodicidad de anillos de crecimiento de dos especies arbóreas en una selva húmeda del norte de Oaxaca, México. Madera y Bosques, 21(3), 55-68.

Pineda-Herrera, E., Valdez-Hernández, J.J., Pérez-Olvera, C.P., \& Dávalos-Sotelo, R. (2015b). Fenología, crecimiento en diámetro y periodicidad de Hura polyandra en Costa Grande, Guerrero, México. Botanical Sciences, 93(4), 741-753.

Pommerening, A., \& Muszta A. (2016). Relative plant growth revisited: Towards a mathematical standardisation of separate approaches. Ecological Modelling, 320, 383-392. DOI: 10.1016/j. ecolmodel.2015.10.015.

Pompa-García, M., De los Santos-Posadas, M.H., ZepedaBautista, M.E., \& Corral-Rivas, J.J. (2011). Un modelo dendrométrico para estimación del diámetro normal a partir de las dimensiones del tocón. Agrociencia, 45, 379-387.

Prodan, M., Peters, R., Cox, F., \& Real, P. (1997). Mensura Forestal. Serie Investigación y Educación de Desarrollo Sostenible. Costa Rica: Instituto Interamericano de Cooperación para la Agricultura.

Purata, S.E., Gerez, P., \& Peters, C.M. (2016). Manual para el monitoreo comunitario del crecimiento de árboles. México: People and Plants International (PPI) - United States Agency for International Development (USAID) - The Nature Conservancy (TNC) - Alianza México REDD+.

Reich, P.B., \& Borchert, R. (1982). Phenology and ecophysiology of the tropical tree, Tabebuia neochrysantha (Bignoniaceae). Ecology, 63, 294-299. DOI: $10.2307 / 1938945$.

Ricker, M., \& Del Río, R. (2004). Projecting diameter growth in tropical trees: A new modeling approach. Forest Science, 50(2), 213-224.

Rojas, R.F. (2006). Plantaciones Forestales. Costa Rica: Editorial Universidad Estatal a Distancia.

Rojas, R.F., \& Torres, C.G. (2008). Árboles del Valle Central de Costa Rica: reproducción. Revista Forestal Mesoamericana Kurú, 5(14), 1-3.

Santos, J.V., Carreón, M.S., \& Nelson, K.C. (1998). La organización de ejidos productores forestales de la zona maya. México: Editorial Futura. 
SAS Institute Inc. (2014). 13.2 User's Guide. EE.UU.: SAS Institute Inc.

Schmidt, L., \& Jøker, D. (2000). Swietenia macrophylla. Københavns Universitet. Seed Leaflet, (30), 1-2.

SEMARNAT [Secretaría de Medio Ambiente y Recursos Naturales] (2014). Inventario Estatal Forestal y de Suelos 2013. México: SEMARNAT.

SEMARNAT [Secretaría de Medio Ambiente y Recursos Naturales] (2016). Anuario Estadístico de la Producción Forestal 2016. México: SEMARNAT- Dirección General de Gestión Forestal y de Suelos.

Smith, T.M., Leemans, R., \& Shugart, H.H. (1992). Sensitivity of terrestrial carbon storage to $\mathrm{CO} 2$-induced climate change: Comparison of four scenarios based on general circulation models. Climate Change, 21, $367-384$.

Shono, K., \& Snook, L.K. (2006). Growth of big-leaf mahogany (Swietenia macrophylla) in natural forests in Belize. Journal of Tropical Forest Science, 18(1), 66-73.

Snook, L.K. (2000). Regeneración y crecimiento de caoba (Swietenia macrophylla) en selvas de Quintana Roo. Ciencia Forestal en México, 25(87), 59-76.
Snook, L.K. (2003). Regeneration, growth, and sustainability of mahogany in Mexico's Yucatan forests. En A. Lugo, J.C. Figueroa-Colón, \& M. Alayón (Eds.), Bigleaf mahogany: Genetics, Ecology, and Management (pp. 169-192). EE.UU.: Springer-Verlag.

Synnott, T.J. (2009). La caoba en la península de Yucatán. Reporte final: diagnóstico de los sistemas de silvicultura de caoba en los corredores de la península de Yucatán (Reporte técnico). México: Comisión Nacional para la Biodiversidad.

Van Laar, A., \& Akça, A. (1997). Forest mensuration. Germany: Cuvillier Verlag.

Van Rheenen, H.M.P.J.B., Boot, R.G.A., Werger, M.J.A., \& Ulloa, M.U. (2004). Regeneration of timber trees in a logged tropical forest in north Bolivia. Forest Ecology \& Management, 200, 39-48.

Vester, H.F.M., \& Navarro-Martínez, M.A. (2007). Árboles maderables de Quintana Roo. México: COQCYT CONABIO - ECOSUR.

Vibrans, A.C., Moser P., Zimermann, O.L., \& Mazaneuro, J.P. (2015). Height-diameter models for three subtropical forest types in Southern, Brazil. Ciencia e Agrotecnologia, 39(3), 205-215. 